
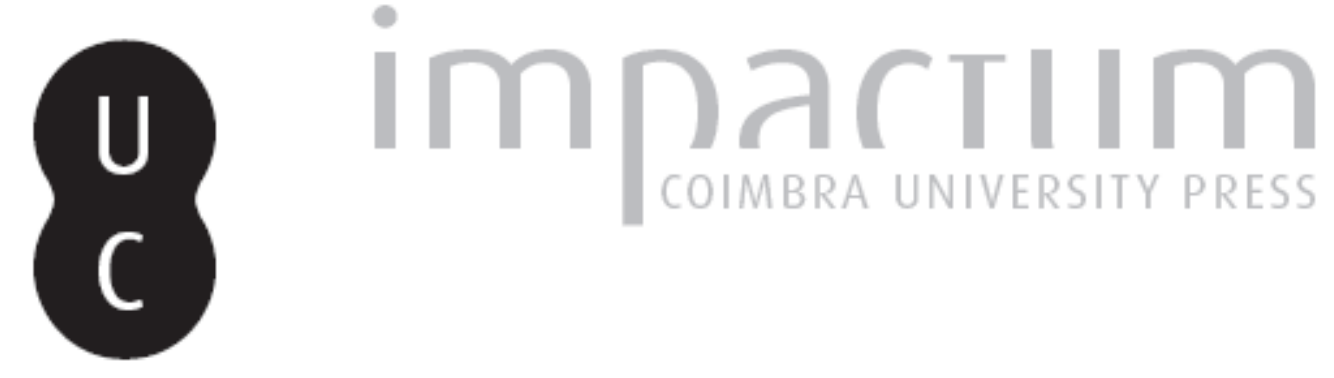

\title{
Mapeamento do risco de ravinamento na área de proteção ambiental Fernão Dias, Sudeste do Brasil, a partir de lógica de decisão Fuzzy
}

Autor(es): $\quad$ Ferreira, Marcos César; Garófalo, Danilo Trovó; Ferreira, Marta Marujo

Publicado por: Associação Portuguesa de Riscos, Prevenção e Segurança; Imprensa

URL

persistente:

da Universidade de Coimbra

DOI: DOl:http://dx.doi.org/10.14195/1647-7723_22_5

Accessed : $\quad$ 26-Apr-2023 08:59:30

A navegação consulta e descarregamento dos títulos inseridos nas Bibliotecas Digitais UC Digitalis, UC Pombalina e UC Impactum, pressupõem a aceitação plena e sem reservas dos Termos e Condições de Uso destas Bibliotecas Digitais, disponíveis em https://digitalis.uc.pt/pt-pt/termos.

Conforme exposto nos referidos Termos e Condições de Uso, o descarregamento de títulos de acesso restrito requer uma licença válida de autorização devendo o utilizador aceder ao(s) documento(s) a partir de um endereço de IP da instituição detentora da supramencionada licença.

Ao utilizador é apenas permitido o descarregamento para uso pessoal, pelo que o emprego do(s) título(s) descarregado(s) para outro fim, designadamente comercial, carece de autorização do respetivo autor ou editor da obra.

Na medida em que todas as obras da UC Digitalis se encontram protegidas pelo Código do Direito de Autor e Direitos Conexos e demais legislação aplicável, toda a cópia, parcial ou total, deste documento, nos casos em que é legalmente admitida, deverá conter ou fazer-se acompanhar por este aviso. 


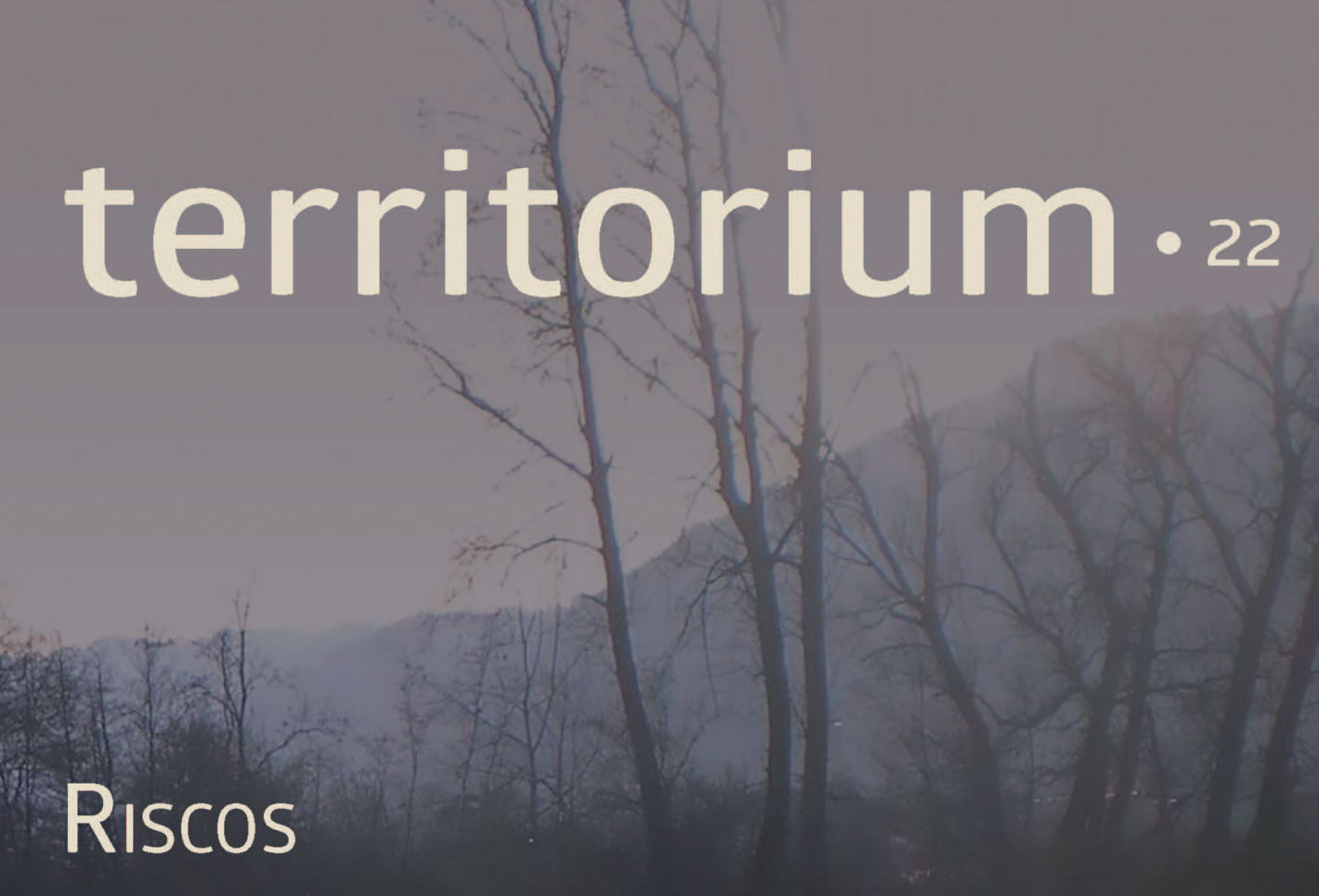

\section{TERRITÓRIOS DE CONVERGÊNCIA}

- Imprensa da Universidade de Coimbra Associação Portuguesa de:Riscos, Prevenção e Segurança 


\title{
DINÂMICA HIDROLÓGICA E EROSIVA EM AGRICULTURA TRADICIONAL DE CORTE E QUEIMA NO BIOMA DE MATA ATLÂNTICA (RJ) - BRASIL*
}

HYDROLOGICAL AND EROSIVE DYNAMICS IN TRADITIONAL SLASH-AND-BURN FARMING METHODS IN THE ATLANTIC FOREST BIOME (RJ) - BRAZIL

\author{
Ana Valéria Freire Allemão Bertolino \\ Departamento de Geografia / Universidade do Estado do Rio de Janeiro \\ anabertolino@uol.com.br \\ Isabel Linhares Pereira Soares \\ Departamento de Geografia / Universidade do Estado do Rio de Janeiro \\ linharesgeo@hotmail.com \\ Lúcio Cunha \\ Departamento de Geografia e CEGOT/ Universidade de Coimbra, Portugal \\ lucio@uc.pt
}

\section{RESUMO}

O objetivo do trabalho é analisar as características hidro-erosivas em sistemas de manejos distintos: sem cobertura (SC) e sistema abandonado de corte/queima de 3-5 anos (PO). Os resultados demonstram que o sistema SC possui valores de potenciais matriciais próximo à saturação, enquanto que o sistema PO possui variações constantes de potenciais matriciais e baixas taxas de erosão, demonstrando uma drenagem eficiente quando comparado ao sistema SC.

Palavras-chave: Pousio, queimada, comportamento hidrológico, erosão.

\section{ABSTRACT}

The aim of the present work is to analyze the hydro-erosive characteristics of different management systems: uncovered soil system and abandoned slash-and-burn system of 3-5 years. Results have shown that the uncovered soil system presents matric potential value close to saturation while the slash-and-burn system demonstrates a frequent variation of the matric potential value and a low erosion rates, indicating an effective drainage system when compared to uncovered soil system.

Keywords: Fallow system, burning, hydrological behavior, erosion.

\section{RESUMEN}

Dinámica hidrológica y erosiva en la agricultura tradicional de corte y quema en el bioma del bosque atlántico (RJ) - Brasil - El objetivo del trabajo es analizar las características de erosión hídrica en sistemas diferentes de manejo: sin cobertura (SC) y sistema abandonado de corte/quema de 3-5 años (PO). Los resultados muestran que el sistema SC presenta valores de potenciales matriciales cercanos a la saturación, mientras que en el sistema PO se observan variaciones constantes de potenciales matriciales y bajas tasas de erosión, lo que demuestra un sistema de drenaje eficaz en comparación con el SC.

Palabras clave: Berbecho, quemada, comportamiento hidrologico, erosión.

RÉSUMÉ

Dynamique hydrologique et érosive de l'agriculture de coupe et sur brûlis dans le biome de la forêt atlantique (RJ) - Brésil - L'objectif de cette publication est d'analyser les caractéristiques hydro-érosives de différentes techniques de gestion des terres: le système sans couverture, et le système de coupe/sur brûlis abandonné entre 3-5 ans. Les résultats montrent que la gestion dans le système sans couverture a un potentiel de matrice proche à la saturation tandis que le système de coupe/sur brûlis abandonné a un potentiel de matrice variable avec des taux d'érosion assez faibles, démontrant un système de drainage efficace en comparaison au système sans couverture.

Mots-clé: Jachère, agriculture sur brûlis, comportement hydrologique, érosion.

* O texto deste artigo corresponde a uma comunicação apresentada no III Congresso Internacional, I Simpósio Ibero-Americano e VIII Encontro Nacional de Riscos, tendo sido submetido em 30-01-2015, sujeito a revisão por pares a 30-04-2015 e aceite para publicação em 24-07-2015.

Este artigo é parte integrante da Revista Territorium, n. ${ }^{\circ} 22,2015,{ }^{\circ}$ RIscos, ISSN: 0872-8941. 


\section{Introdução}

O sistema agrícola de corte e queima (shifting agriculture ou slash-and-burn) é o sistema agrícola mais extensivo do mundo, ocorre predominantemente nos Trópicos e tem grande importância na subsistência de cerca de 250 a 500 milhões de pessoas ao redor do mundo (FAO, 1985; N. C. Brady, 1996; N. Pedroso Júnior et al., 2008). Este sistema é praticado nas áreas florestadas, principalmente nas regiões tropicais (O. Valverde, 1968; D. G. McGrath, 1987; O. Mertz et al., 2009; A. V. F. A. Bertolino e L. C. Bertolino, 2010; C. Padoch e M. Pinedo-Vasquez, 2010; A. A. R. Filho et al., 2013; R. Nigh e S.A.W. Diemont, 2013) e, provavelmente, é a forma mais antiga da agricultura nas Américas (G. Barker, 2006).

O sistema agrícola de corte e queima envolve uma gama de técnicas que denotam seu caráter diversificado em diferentes partes do mundo, entretanto tem como característica similar o aproveitamento do capital energético da floresta em recomposição. Mesmo com muitas variantes, as características essenciais do padrão de cultivo nesses sistemas são a utilização do corte da vegetação, uso do fogo e períodos de pousio da terra após a colheita. Apesar deste sistema constituir um manejo criticado por diversos autores (E. N. Chidumayo e L. Kwibisa, 2003; M. A. MacDonald et al., 2000; D. Lawrence et al., 2007), ele auxilia na regeneração das áreas a partir da utilização do pousio, o que atesta a sua sustentabilidade quando praticado tradicionalmente ( $R$. Nye e P. H. Greenland, 1960; J. Frizano et al., 2003; C. M. Johnson et al., 2001; R. Nigh e S. A. W. Diemont, 2013).

A agricultura migratória está relacionada com qualquer sistema agrícola no qual os campos são desmatados e cultivados por períodos curtos, seguidos de um período de descanso dos solos (N. Pedroso Júnior et al., 2008, A. A. R. Filho et al., 2013). É entendida também como uma estratégia de manejo dos recursos mediante a qual os agricultores saem de uma área para outra a fim de aproveitar a fertilidade do novo terreno (D. G. McGrath, 1987; N. Pedroso Júnior et al., 2009).

Algumas das suas características gerais são: o reduzido tamanho da área plantada, a duração do pousio, que se estende por 3 a 5 anos, e a baixa intensidade de comercialização; os métodos de cultivo, baseados em força humana e animal; as técnicas de cultivo, que são restritas e com pouco uso de instrumentos e mecanização; e a fertilidade do solo, mantida com aportes de esterco animal e pelos nutrientes provenientes das cinzas e da decomposição vegetal.

Segundo P. A. Sanchez et al. (2005) a shifting agriculture e slash-and-burn são dois sistemas distintos que apresentam características peculiares. 0 primeiro sistema está associado a um sistema de rotação tradicional, com longos períodos de pousio. 0 segundo está associado a curtos períodos de pousio. Neste estudo será utilizado o conceito de slash-and-burn adotado pelos autores citados acima.

Um dos problemas relacionados com o sistema de slashand-burn é a redução do tempo de pousio (O. K. Borgaard et al., 2003; D. Lawrence, 2007; E. R. Styger et al., 2007). Outro problema está centrado na utilização de fogo, pois este pode trazer modificações nas propriedades do solo (G. Certini, 2005; R.A. Shakesby e S.H. Doerr, 2006; J. Mataix-Solera et al., 2011), aumento da repelência da água (I. F. DeBano, 2000), intensificação do escoamento superficial (A. Cerdá e T. Lasanta, 2005) e perda de nutrientes (D. Lawrence et al., 2007). Por outro lado, o sistema de slash-and-burn é também analisado por diversos autores que constatam alguns aspectos de melhoria do sistema, tais como: recarga e drenagem dos solos (M. P. Errea et al., 2001), aumento dos macroagregados (ObaleEbanga et al., 2003) e diminuição da erosão e escoamento superficial (E. L. Thomaz, 2013).

O tempo de pousio utilizado pelos agricultores na região de São Pedro da Serra (Nova Friburgo - RJ) vem diminuindo (com duração máxima de 3-5 anos) para que as árvores presentes na regeneração vegetal não ultrapassem $5 \mathrm{~cm}$ de diâmetro de tronco (DAP), pois este é um dos parâmetros utilizados pela legislação para que a área seja considerada intocável e de preservação ambiental (A. V. F. A. Bertolino e L. C. Bertolino, 2010). A cada três ou quatro ciclos de plantio, a terra fica parada por 3 ou 5 anos. O preparo do terreno para o início do cultivo se dá da seguinte maneira: limpeza do terreno, com a queimada dos resíduos da lavoura anterior, seguida da aração; esta é feita conforme as curvas de nível e utilizando arado de boi. O período de corte é feito normalmente no inverno (junho-julho-agosto) e a queima ocorre na primavera (setembro-outubro). Após o corte da vegetação, esta é posta a secar, seguida da queima do material.

Em ambientes tropicais montanhosos são poucos os estudos voltados para as questões e repercussões da agricultura de corte/queima com pousio na hidrologia superficial. 0 principal objetivo deste estudo é comparar sistemas de manejo: a) sem cobertura vegetal; e b) área abandonada de corte, queima e pousio de 3-5 anos e suas repercussões na dinâmica hidrológica superficial em parcelas experimentais localizadas em São Pedro da Serra, município de Nova Friburgo - Sudeste do Brasil.

\section{Área de estudo}

O estudo foi desenvolvido no Campus Experimental de São Pedro da Serra, distrito de Nova Friburgo, localizado na Serra do Mar, Brasil nas coordenadas geográficas $22^{\circ}$ 18' 52.01" S e $42^{\circ} 20^{\prime} 04.22$ " 0 , que está inserido no Bioma de Mata Atlântica e faz parte da Área de Proteção Ambiental de Macaé de Cima (fig. 1). 

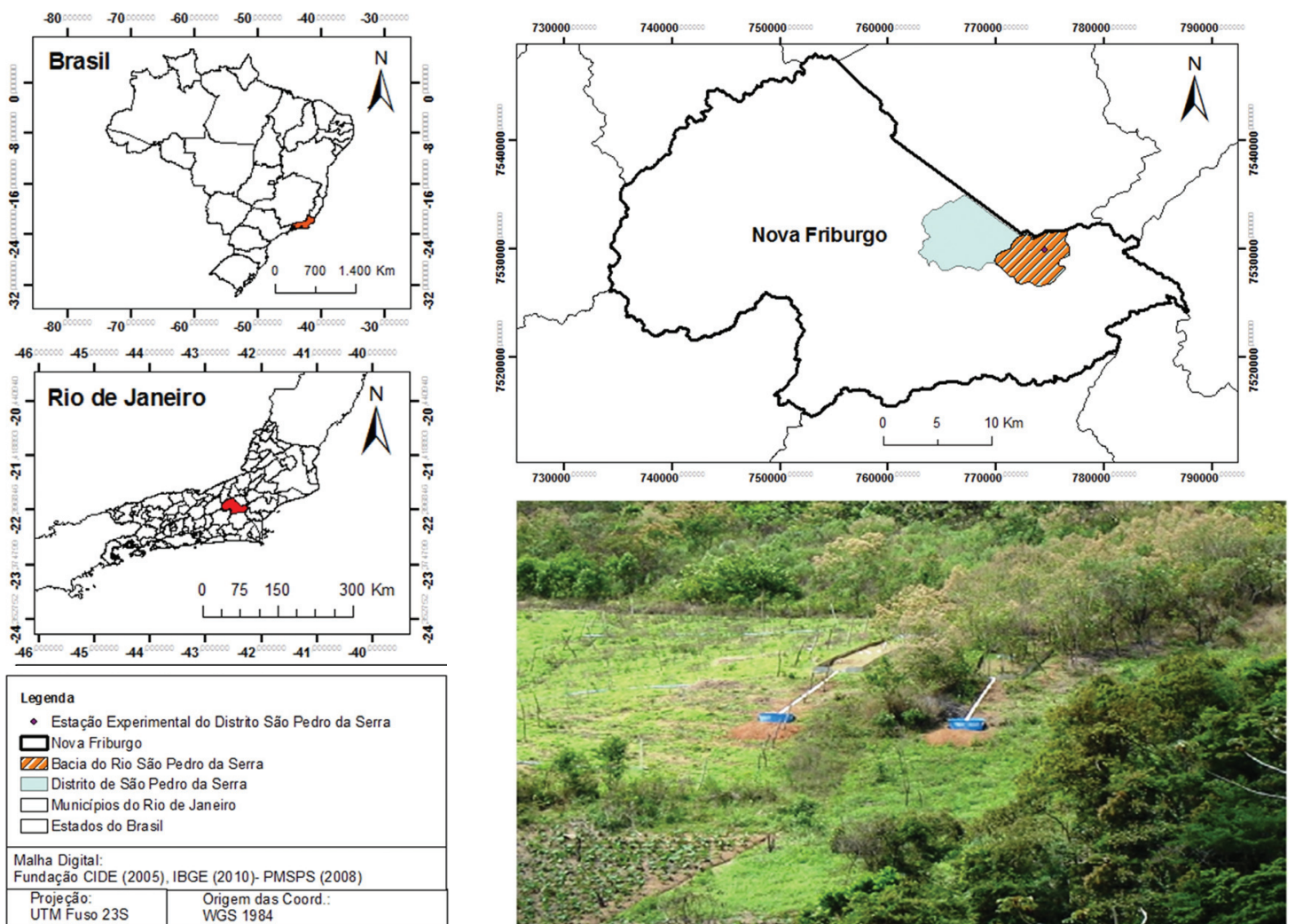

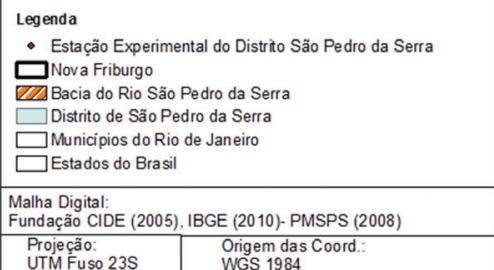

Fig. 1 - Mapa de localização e visão geral da Estação Experimental do distrito São Pedro da Serra, Rio de Janeiro - Brasil.

Fig. 1 - Localization map and overview of the Experimental Station of São Pedro da Serra, Rio de Janeiro - Brazil.

A agricultura tradicional de corte/queima/pousio tem sido feita nesta área há mais de 100 anos. Nas últimas décadas, os cultivos mais utilizados são de aipim, batata doce, batata baroa, couve-flor, inhame, feijão, repolho, tomate e pimentão, variando conforme a época do ano.

A região apresenta um clima Tropical de Altitude, caracterizado por verões quentes e chuvosos e invernos amenos e secos. Segundo a classificiação de C. W. Tornthwaite e J. R. Mather, (1955) é de tipo A - superúmido e tipo $B^{\prime}$ - Mesotérmico. A temperatura média varia entre 20,3 a $21,2^{\circ} \mathrm{C}$ no período do verão (trimestre dezembro-fevereiro), e entre 14,1 e $15,3^{\circ} \mathrm{C}$ no inverno (junho-agosto). A precipitação média anual é de $1.279,8 \mathrm{~mm}$, sendo os meses mais chuvosos de novembro a março, e os meses mais secos de maio a agosto, marcando o período sazonal de chuvas (verão) e secas (inverno), respectivamente (INMET, 2014).

O balanço hídrico da região analisada, com base na classificação de Tornthwaite e Mather (1955), mostra que há uma estação de deficiência hídrica centrada nos meses de maio a setembro, em que o déficit de água do solo é de $20,8 \mathrm{~mm}$. Os meses de novembro a abril apresentam excedente hídrico médio total de 438,6 mm.

A área de estudo está inserida na Serra do Mar, pertencente à Faixa Móvel Ribeira, que, estruturalmente, compreende um complexo cinturão de dobramentos e empurrões de trend NE-SW gerados na borda sul/ sudeste do Cráton de São Francisco, a partir da colisão deste com outras placas e/ou microplacas (M. Heilbron et al., 2004). Considerando a seção que inclui a área da APA Estadual de Macaé de Cima, a Faixa Ribeira é compartimentada em apenas uma unidade tectonoestratigráfica: Terreno Oriental. Encontra-se na unidade geomorfológica dos Planaltos e Serras do Leste-Sudeste (J. Ross, 1985). Nesta escala, a Área de Proteção Ambiental se insere em formações planálticas sobre cinturões orogenéticos (Faixa Móvel Ribeira) e compreende dois domínios geomorfológicos: escarpas serranas e domínio montanhoso (M. E. Dantas, 2000).

Na sub-bacia do rio São Pedro, onde está inserida a Estação Experimental de Pesquisa de Erosão (EEPE/ SPS), há um predomínio da declividade acima de $26 \%$ e a elevação encontra-se em sua maioria nas cotas entre $880 \mathrm{~m}$ a $1210 \mathrm{~m}$. As principais classes de solos que ocorrem são os Neossolos Litólicos, inseridos em relevo montanhoso/escarpado, Latossolos Vermelho-Amarelos e Cambissolos Háplicos (A. Carvalho Filho et al., 2000), formados a partir de gnaisses biotíticos, kinzigitos, e depósitos colúvio-aluvionares.

Os solos nas parcelas de erosão correspondem a Cambissolos e apresentam uma classe de textura denominada de solo franco, com $46 \%$ de areia, $28 \%$ de 
argila e $26 \%$ de silte. Os solos são classificados como fortemente ácidos ( $\mathrm{pH}$ que varia de 4,0 a 4,7) com valores de carbono que variam de $1,6 \%$ e $1,1 \%$, respectivamente.

$\mathrm{Na}$ área com pousio de 3 a 5 anos são encontrados valores de condutividade hidráulica da ordem de 4,23 × $10^{-3} \mathrm{em}$ $15 \mathrm{~cm}$ e $1,76 \times 10^{-2}$ em $30 \mathrm{~cm}$, classificados em média e alta permeabilidade. 0 sistema sem cobertura apresenta $3,17 \times 10^{-3}$ em $15 \mathrm{~cm}$ e 7,06 × $10^{-4} \mathrm{em} 30 \mathrm{~cm}$, classificados em média e baixa permeabilidade (G. Merat, 2014).

Segundo K. K. S. Costa (2012) a área com pousio de 3 a 5 anos apresenta um estrato arbóreo mais homogêneo, uma vez que a maioria são espécies heliófilas, exigentes de luminosidade. Foram encontradas 20 espécies dentre as quais: Machaerium stipitatum(DC.) Vogel, Vernonia polyanthes Less., Croton floribundus Spreng., Persea americana Mill. e Psychotria vellosiana Benth. A espécie Machaerium stipitatum é a que apresenta o maior número de indivíduos, mas a que demonstra maior adaptabilidade ao ambiente, apresentando as maiores alturas e diâmetros é Vernonia polyanthes. Em relação à estrutura da vegetação, a autora constatou valores de área basal em áreas com pousio de 3-5 anos, com pousio de 10-12 anos e floresta de forma crescente: $3,1 \mathrm{~m}^{2} /$ ha, $11,9 \mathrm{~m}^{2} /$ ha e $18,4 \mathrm{~m}^{2} / \mathrm{ha}$. A área de floresta apresenta diâmetro a altura do peito médio de $8,44 \mathrm{~cm}$, o sistema com pousio de 10 a 12 anos de $7,71 \mathrm{~cm}$ e o sistema com pousio de $3-5$ anos de $6,36 \mathrm{~cm}$.

\section{Materiais e métodos}

O monitoramento das parcelas de erosão ocorreu no período de dezembro de 2008 a julho de 2011. O estudo foi desenvolvido na Estação Experimental de Pesquisa de Erosão (EEPE/SPS) e foi realizado em um sistema sem cobertura ( $\mathrm{SC}$ ) e num sistema abandonado de corte/queima e com pousio de 3 a 5 anos (PO), no início do pousio.

As parcelas de erosão possuem $10 \times 2 \mathrm{~m}$, totalizando $20 \mathrm{~m}^{2}$. As parcelas foram delimitadas através de chapas de ferro galvanizadas, com espessura de 2 a $4 \mathrm{~mm}$ e $60 \mathrm{~cm}$ de altura $(40 \mathrm{~cm}$ enterrados no solo e $20 \mathrm{~cm}$ aparentes, para impedir a entrada e a saída de material por erosão de salpicamento) e estão conectadas a caixas coletoras de $500 \mathrm{l}$, a fim de medir as perdas de água e de solo por erosão superficial. As parcelas foram instaladas na porção média da encosta com uma declividade de $26 \%$ (fig. 2).

O monitoramento do escoamento superficial foi realizado diariamente às 07:00h e o escoamento superficial das parcelas que apresentaram uma alíquota mínima de 1 litro de água foi coletado com a mesma frequência. Os procedimentos para coleta e quantificação do escoamento superficial e dos sedimentos foram obtidos conforme a metodologia de N. W. Hudson (1993).
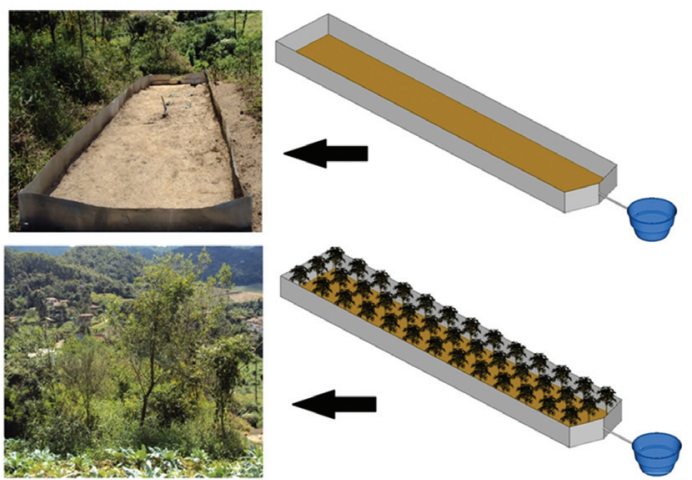

Fig. 2 - Visão geral das duas parcelas de erosão $\left(20 m^{2}\right)$ : sem cobertura vegetal (SC) e sistema abandonado de corte/queima e com pousio de 3 a 5 anos (PO).

Fig. 2 - Overview of the two erosion plots $\left(20 \mathrm{~m}^{2}\right)$ : Uncovered Soil System (USS) and Abandoned Slash-and-burn System of 3-5 years.

A dinâmica hidrológica do solo foi caracterizada pelo monitoramento dos potenciais matriciais da água no solo a 15 e $30 \mathrm{~cm}$ de profundidade, durante o período de 2008-2011.

Em cada parcela de erosão foram colocados blocos de matriz granular (GMS's), dois por cada profundidade, instalados no meio de cada parcela (fig. 3). Os potenciais matriciais foram monitorados com o Watermark 200SS sensors (Irrometer Co., Riverside, CA, USA). O sensor Watermark, chamado de bloco de matriz granular (GMS), mede o potencial matricial da água no solo indiretamente baseado na resistência elétrica ( $E$. P. Eldredge et al., 1993; C. C. Shock et al., 1998; C. C. Shock, 2003). Foram utilizados um total de 8 blocos de matriz granular instalados em duas parcelas de erosão e medidos diariamente às 7:00 $\mathrm{h}$.

A precipitação foi monitorada através de uma Estação Meteorológica Automatizada THIES TLX-MET durante todo o período. Os dados foram coletados diariamente

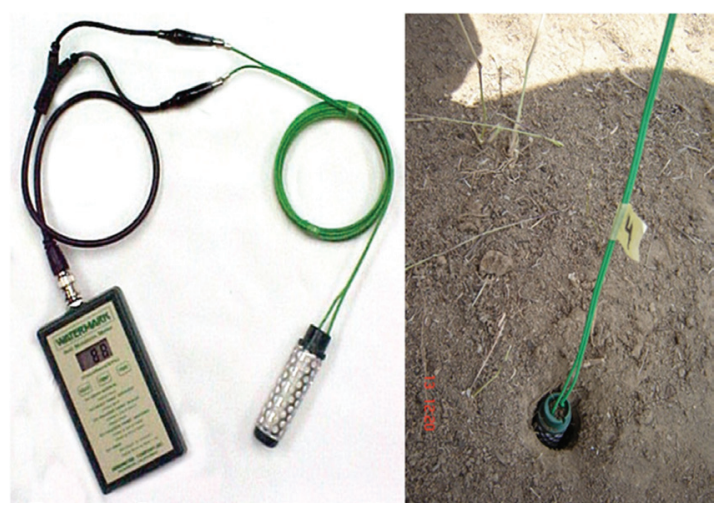

Fig. 3 - Medidor portátil Watermark (Fonte: Irrometer Company, 2008) e instalação do bloco de matriz granular.

Fig 3 - Portable Watermark Meter (Source: Irrometer Company, 2008) and installation of the granular matrix block. 
a cada 10 minutos, sendo que a leitura era realizada às $07: 00 \mathrm{~h}$. A estação foi instalada perto das parcelas de erosão.

Para medição da temperatura do solo, de modo a permitir calibrar o leitor do GMS, foram utilizados geotermômetros Incoterm ${ }^{\circledR}$ com haste rígida de $11 \mathrm{~cm}$. As temperaturas dos geotermômetros inatermark 200SS elétrons foram também registradas diariamente às 07:00h da manhã.

\section{Resultados e discussão}

Os valores de precipitação diária foram registrados durante os anos de 2006 a 2011, com totais pluviométricos de 1.707, 1.393, 1.989, 2.050, 1.802 e $2.014 \mathrm{~mm}$, respectivamente, apresentando uma média de 1.826 $\mathrm{mm}$ (fig. 4).

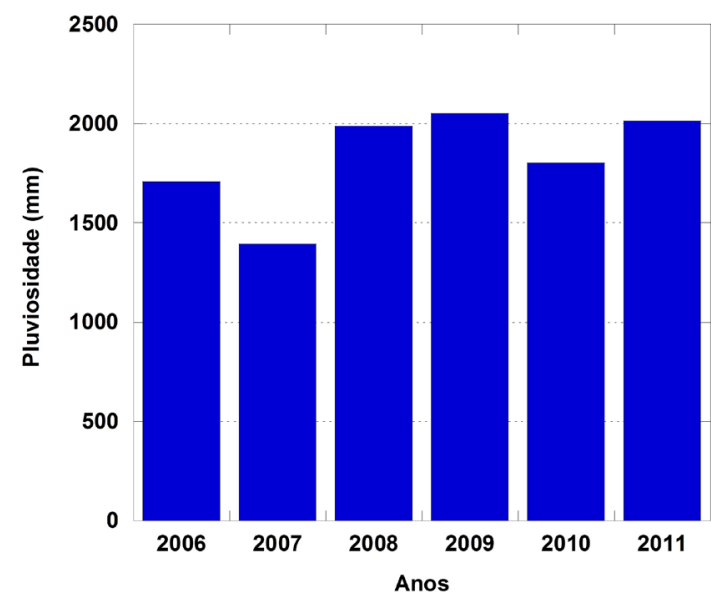

Fig. 4 - Precipitação anual da Estação Climatológica Convencional (2006-2011) de São Pedro da Serra, Rio de Janeiro - Brasil.

Fig. 4 - Total annual precipitation of Conventional Climatological Station (2006-2011) of São Pedro da Serra, Rio de Janeiro - Brazil.

$\mathrm{Na}$ comparação das médias mensais da Estação Convencional com as Normais Climatológicas de 1961 a 1990, verifica-se que os índices pluviométricos da estação chuvosa, principalmente em novembro e dezembro, tiveram um aumento expressivo. Os meses de fevereiro e maio foram os únicos que mantiveram suas médias. A partir da média mensal de todos os anos (2006 a 2011) observam-se dois períodos distintos: um período úmido (novembro a abril); de maior ocorrência de chuvas; e um período seco (maio a outubro), de menor ocorrência (fig. 5).

Em relação às condições de recarga e drenagem do solo no período de 2008 a 2011, verificou-se que o sistema abandonado de corte/queima com pousio de 3-5 anos demonstrou drenagem mais eficiente $(-70 \mathrm{kPa})$ quando comparado ao sistema sem cobertura vegetal $(-40 \mathrm{kPa})$,

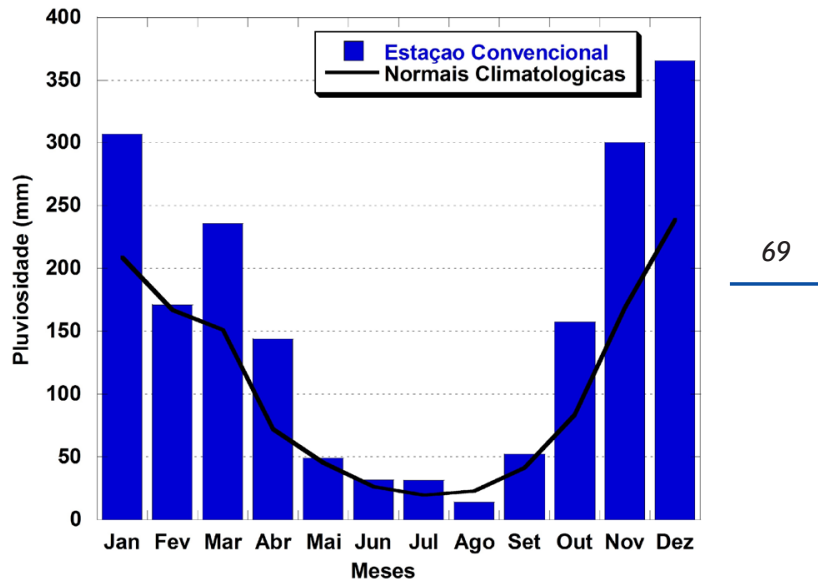

Fig. 5 - Média mensal de pluviosidade da Estação Climatológica Convencional (2006-2011) de São Pedro da Serra, Rio de Janeiro - Brasil e das Normais Climatológicas (1961-1990).

Fig. 5 - Average monthly rainfall of the Conventional Climatological Station (2006-2011) and the Climate Normals (1961-1990) of São Pedro da Serra, Rio de Janeiro - Brazil.

tendo em vista o comportamento pluviométrico da região. 0 mesmo é verificado na média geral do período úmido, em que o SC apresentou $-21 \mathrm{kPa}$ e $\mathrm{PO}-49$ $\mathrm{kPa}$; e para o período seco, em que o SC apresentou -64 kPa e o PO -98 kPa (QUAdRo I). Durante o período úmido são menores as amplitudes de variação entre os sistemas SC e PO. No período seco ocorre o contrário ou seja há um aumento das diferenças dos potenciais matriciais demonstrando a influência do manejo e uso na drenagem do solo.

QUADRo I - Média do potencial matricial no período geral e nos períodos seco e úmido.

TABLE I - Average of the matric potential in the overall period and in the dry and wet periods.

\begin{tabular}{cccc}
\hline Sistemas & $\begin{array}{c}\text { Período Geral } \\
(2008-2011)\end{array}$ & $\begin{array}{c}\text { Período } \\
\text { Seco }\end{array}$ & $\begin{array}{c}\text { Período } \\
\text { Úmido }\end{array}$ \\
\hline SC & $-40 \mathrm{kPa}$ & $-64 \mathrm{kPa}$ & $-21 \mathrm{kPa}$ \\
\hline $\mathrm{PO}$ & $-70 \mathrm{kPa}$ & $-98 \mathrm{kPa}$ & $-49 \mathrm{kPa}$ \\
\hline
\end{tabular}

Observa-se que as maiores médias dos potenciais matriciais da água no solo (valores mais próximos de 0 $\mathrm{kPa}$ ) se concentram nos meses de janeiro, novembro e dezembro nos dois sistemas, o que condiz com os meses mais chuvosos da região. Porém, o sistema sem cobertura vegetal (SC) apresenta valores ainda mais próximos da saturação quando comparado ao sistema abandonado de corte/queima e pousio (PO) (fig. 6 e fig. 7).

Observa-se que no mês de abril, período em que houve uma diminuição do índice pluviométrico, o sistema SC apresenta valores ainda maiores $(-9 \mathrm{kPa})$, enquanto no sistema $\mathrm{PO}$ esse valor diminui (-54 kPa), acompanhando 


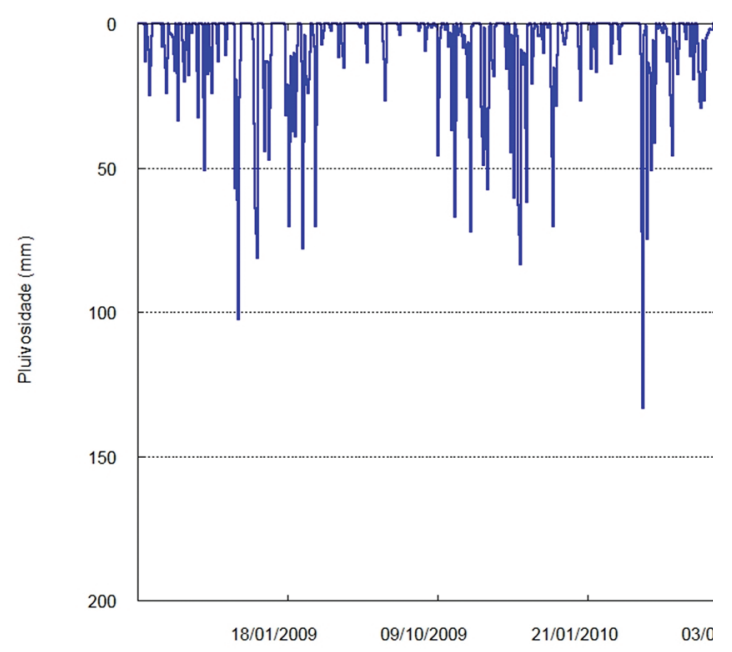

Fig. 6 - Precipitação diária de outubro de 2008 a julho de 2011 - São Pedro da Serra - Brasil.

Fig. 6 - Daily precipitation from October, 2008 to July, 2011São Pedro da Serra - Brazil.

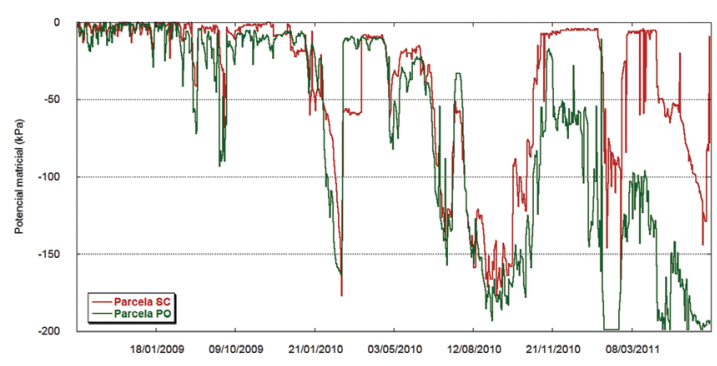

Fig. 7 - Potencial matricial diário de outubro de 2008 a julho de 2011- São Pedro da Serra - Brasil.

Fig. 7 - Daily Matric Potential from October, 2008 to July, 2011 - São Pedro da Serra - Brazil.

o índice pluviométrico. Isso demonstra a dificuldade de drenagem da umidade antecedente no sistema SC. Logo após um período seco, quando se inicia o período de maior ocorrência de chuvas (mês de outubro), o sistema de PO só vai apresentar valores maiores alguns dias depois do sistema sem cobertura, pois, para além da intercepção das chuvas pela vegetação, sofre influência do período de menor concentração de chuvas.

As modificações do uso do solo com a retirada da cobertura vegetal contribuem para a modificação do ciclo hidrológico, caracterizando o desenvolvimento de condições favoráveis a um maior desenvolvimento de processos erosivos por diferentes mecanismos e, consequentemente, contribuindo para um aumento das descargas líquidas e sólidas nos canais fluviais.

M.V.S. Chaves (2009) avaliou na mesma área de estudo a interceptação de diferentes sistemas: a) pousio de 3-5 anos, b) pousio de 10-12 anos e c) floresta de 50-
70 anos. 0 autor constatou ao longo de três anos que a área de floresta apresentou os maiores percentuais de interceptação ( $28 \%$, 30\% e 25\%). No pousio de $10-12$ anos foram determinados valores de $32 \%, 21 \%$ e $22 \%$, enquanto o pousio de 3-5 anos, de modo geral, os menores índices de interceptação (18\%, 20\% e 19\%). Isto ocorreu devido este sistema apresentar um dossel vegetal pouco desenvolvido em comparação aos outros tratamentos, logo tende a ter uma interceptação inferior aos demais sistemas.

Apesar de ocorrerem interceptações baixas na área de PO (em torno de 20\%), o sistema PO apresentou valores de potenciais matriciais sempre menores em relação ao sistema SC, o que demonstra que o PO apresenta uma drenagem mais expressiva do que o sistema SC, comprovando que a regeneração do sistema com pousio influencia claramente na dinâmica hidrológica.

M. P. Errea et al. (2001) verificaram mudanças no conteúdo de umidade em solos cultivados, abandonados (pousio), queimados (queimado 1 e queimado 2) e com cobertura vegetal (30 anos) na Espanha. Os autores constataram a partir do uso de reflectômetro do domínio do tempo (TDR`s), durante dois anos, que no período úmido as mudanças espaciais de umidade entre os diferentes usos eram reduzidas e que durante os períodos secos, ocorria o contrário. A área abandonada (pousio) apresentou um conteúdo médio de umidade de $23 \%$, a área com cobertura vegetal de 30 anos de $35 \%$, a gramínea e o solo desnudo de $31 \%$ e os solos manejados e queimados apresentaram valores intermédios. 0 menor conteúdo de umidade das áreas abandonadas (pousio) foi relacionado com a sucessão da vegetação que altera a dinâmica da umidade do solo. Já nas áreas de solo desnudo, o maior conteúdo de umidade está relacionado com o desenvolvimento de uma crosta no período seco, que induz uma redução da taxa de evaporação.

Com a finalidade de demonstrar o papel desenvolvido pelo sistema abandonado de corte/queima e pousio (PO) nos potenciais matriciais da água no solo, foram isolados/individualizados dois períodos distintos: um período de maior ocorrência de chuvas, registrado desde o início do monitoramento (01 de outubro de 2010 a 29 de janeiro de 2011), período que se insere nos meses de maior quantidade de chuvas (de novembro a abril), e que abrange os dias 11 e 12 de janeiro de 2011; e o período de menor ocorrência de chuvas registrado desde o início do monitoramento ( 22 de maio a 28 de julho de 2011) (fig. 8).

No período úmido destaca-se o dia 12 de janeiro de 2011, com um total diário de precipitação de quase $200 \mathrm{~mm}$, um pouco mais da metade da chuva esperada para todo o mês. Verifica-se que o sistema SC apresenta pouca variação dos potenciais matriciais da água no solo, principalmente do dia 12 de novembro de 2011 a 23 de janeiro de 2011, variando de $-4 \mathrm{kPa}$ a $-10 \mathrm{kPa}$, enquanto 

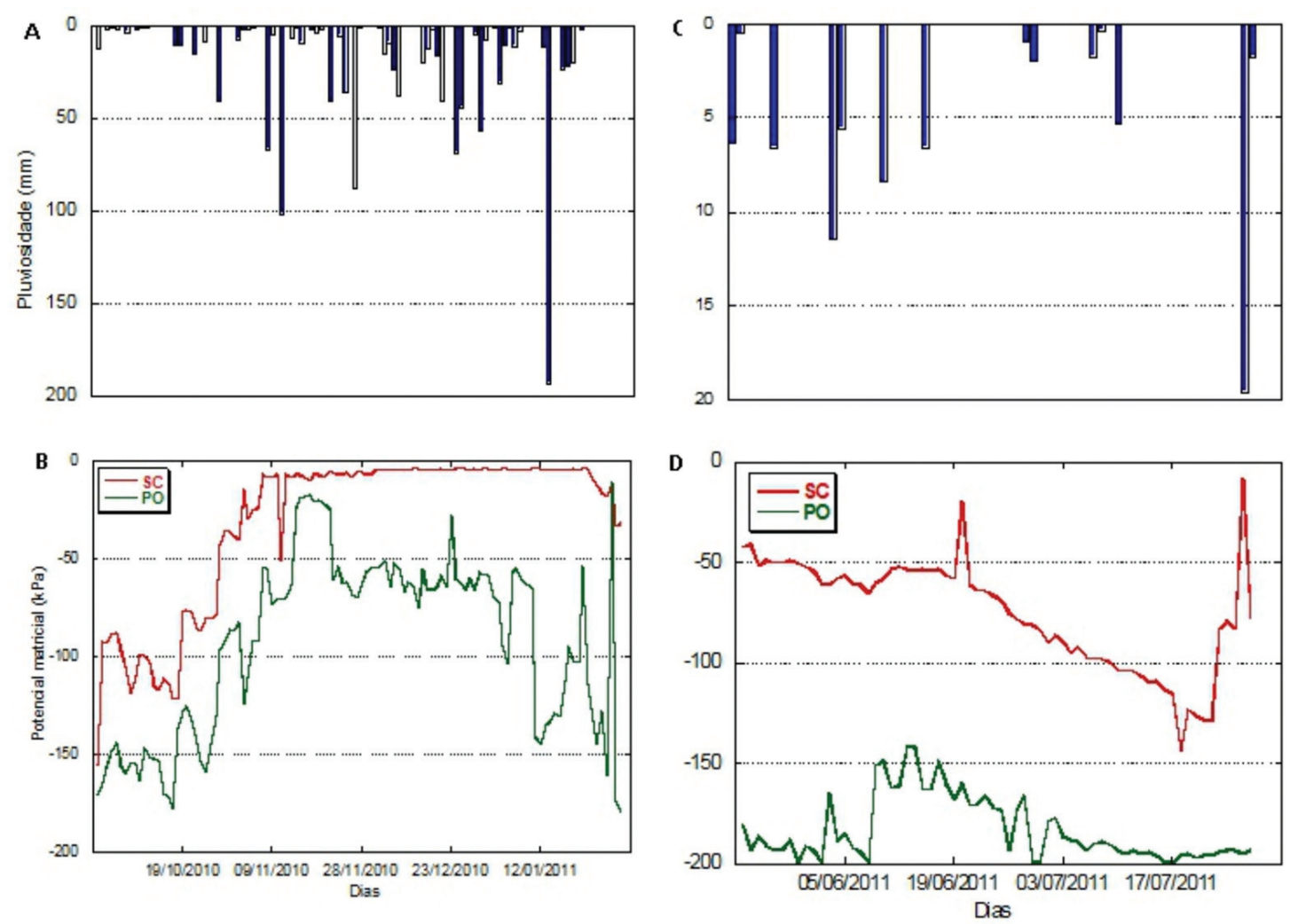

Fig. 8 - A) Precipitação diária de 06 a 17 de janeiro de 2011 B) Potenciais matriciais diários de 06 até 17 de janeiro de 2011 C) Precipitação diária de 30 de maio a 10 de julho de 2011 D) Potenciais matriciais diários de 30 de maio ate 10 de julho de 2011.

Fig. 8 - A) Daily precipitation from January, 06 until 17 - 2011 B) Daily Matric Potential from January, 06 until January, 17 - 2011 C) Daily Precipitation from May, 30 to July 10, 2011 D) Daily Matric Potential from May, 30 until July, 10 July-2011.

o sistema $\mathrm{PO}$, durante o mesmo período, apresentou variações de $-17 \mathrm{kPa}$ a $-145 \mathrm{kPa}$, demostrando uma grande amplitude nos valores.

O sistema SC possui valores superiores ao PO durante grande parte do período, mantendo-se quase invariável mesmo diante de variações pluviométricas, demonstrando uma dificuldade de percolação da água dentro da matriz do solo. Já o sistema PO possui variações constantes em todo o período analisado, demonstrando uma drenagem eficiente quando comparado a SC, mesmo nos períodos de concentração de chuvas.

No período seco de maio a julho de 2011, que se insere nos meses de maior quantidade de chuvas (de novembro a abril), nota-se que ocorreram chuvas pontuais e apenas duas ultrapassaram $10 \mathrm{~mm}$ diários. Após um regime de menor quantidade de chuvas, o sistema de PO drenou de forma eficiente as chuvas do período anterior, variando em -142 kPa a $-199 \mathrm{kPa}$, enquanto SC variou de $-9 \mathrm{kPa}$ a $-144 \mathrm{kPa}$, durante o mesmo episódio pluviométrico. A variação do potencial matricial no sistema de $\mathrm{PO}$, quando relacionada ao SC, é evidenciada na resposta ao evento do dia 24 de julho de 2011 (19,6 mm), em que SC, após dois dias do evento, apresenta valor de $-9 \mathrm{kPa}$, ao mesmo tempo que PO apresenta -195 $\mathrm{kPa}$, um comportamento esperado, levando em consideração o longo período sem chuvas anterior ao dia 24 (14 dias).
O QUADRo II mostra o total de precipitação, escoamento e as perdas de solo mensais para todos os sistemas ao longo do período que vai de dezembro/2008 a julho/2011. Pode-se dizer que em relação ao escoamento superficial em todo o período monitorado o sistema sem cobertura (SC) apresenta os maiores valores, independente da magnitude da precipitação. Em média os valores de escoamento para este tratamento ficam em torno de 1244, $2 \mathrm{~mm}$. O sistema abandonado de PO, por possuir uma vegetação, responsável por proteger o solo da exposição direta dos agentes erosivos, dispersando a energia da água do escoamento superficial, apresentou perdas de água de 0,2 $\mathrm{mm}$ em dezembro de 2008, $15 \mathrm{~mm}$ em janeiro de 2009, 0,06 mm em março de 2009 e 0,3 mm em abril de 2009, totalizando o valor de 15, $2 \mathrm{~mm}$. Nos outros meses até julho de 2011, o sistema PO apresentou perdas de água inferiores a 1 litro, não tendo ocorrido escoamento superficial suficiente para o monitoramento. O sistema PO mostrou-se mais eficaz no controle das perdas de água, quando comparado ao sistema SC, e a presença de resíduos parece ter sido enérgica na redução do escoamento. A elevada resposta dos valores escoados, assim como das perdas de solo para o sistema SC foram agravadas pela falta de cobertura vegetal, o que acarreta o selamento superficial, o aumento da enxurrada e a diminuição da infiltração da água no solo. 
Em algumas situações as práticas de manejo impõem mudanças nas propriedades dos solos que levam em geral a um decréscimo da qualidade física do solo e causam uma variedade de modificações no balanço hídrico (J. A. Vomocil e W. J. Flocker, 1966; B. P. Warkentin, 1971). Os valores de potenciais matriciais, escoamento superficial e erosão no sistema SC demonstram uma tendência do processo de selamento superficial pela destruição dos agregados do solo, em consequência do impacto das gotas de chuva.
O comportamento de SC também é válido para a perda de solo, praticamente com as mesmas características em sua resposta, apresentando valores totais de 29,5 t/ha. Já o sistema PO apresentou um total de perda de solo de apenas 0,0085 t/ha, demonstrando a importância do sistema na regeneração do solo.

O QUADRO III mostra os valores de escoamento superficial e perda de solo de acordo com as fases de regeneração da agricultura de corte e queima (pós-queima/início do

QUADRo II - Pluviosidade e perdas de água e solo mensais de dezembro de 2008 a julho de 2011.

TABLE II - Monthly rainfall and water and soil loss from December, 2008 until July, 2011.

\begin{tabular}{|c|c|c|c|c|c|}
\hline \multirow[t]{2}{*}{ Meses } & \multirow[t]{2}{*}{ Pluviosidade (mm) } & \multicolumn{2}{|c|}{ Escoamento (mm) } & \multicolumn{2}{|c|}{ Perda de solo (t/ha) } \\
\hline & & $\mathrm{SC}$ & PO & SC & PO \\
\hline Dez 2008 & 378,10 & 12 & 0,27 & 0,00948 & 0,0061057 \\
\hline Jan 2009 & 447,90 & 59,40 & 15 & 6,56 & 0,002387 \\
\hline Mar 2009 & 142,60 & 80,30 & 0,06 & 4,809144 & 0,000714 \\
\hline Abr 2009 & 102,10 & 11,40 & 0,36 & 0,581206 & 0,000019 \\
\hline Mai 2009 & 77,30 & 15 & 0 & 0,41592 & 0 \\
\hline Jun 2009 & 54,90 & 2,25 & 0 & 0,1645 & 0 \\
\hline Jul 2009 & 14,60 & 4,20 & 0 & 0,27636 & 0 \\
\hline Set 2009 & 98,70 & 20,75 & 0 & 0,00101493 & 0 \\
\hline Out 2009 & 242,70 & 84,75 & 0 & 2,436 & 0 \\
\hline Nov 2009 & 395,60 & 122,3 & 0 & 3,862768 & 0 \\
\hline Dez 2009 & 473,90 & 49,95 & 0 & 0,594405 & 0 \\
\hline Jan 2010 & 101,10 & 22,70 & 0 & 0,4863 & 0 \\
\hline Fev 2010 & 173,60 & 62,40 & 0 & 0,34498 & 0 \\
\hline Mar 2010 & 343,20 & 58,35 & 0 & 0,489765 & 0 \\
\hline Abr 2010 & 139,30 & 20,05 & 0 & 0,403065 & 0 \\
\hline Mai 2010 & 52,80 & 0,60 & 0 & 0,00864 & 0 \\
\hline Jun 2010 & 27,30 & 0 & 0 & 0 & 0 \\
\hline Jul 2010 & 41,10 & 5,40 & 0 & 0,00864 & 0 \\
\hline Ago 2010 & 22,40 & 0,07 & 0 & 0,000825 & 0 \\
\hline Set 2010 & 19,30 & 0 & 0 & 0 & 0 \\
\hline Out 2010 & 123,70 & 23,45 & 0 & 0,236555 & 0 \\
\hline Nov 2010 & 378,70 & 196,20 & 0 & 2,66532 & 0 \\
\hline Dez 2010 & 185,70 & 170 & 0 & 1,76878 & 0 \\
\hline Jan 2011 & 332,05 & 90,90 & 0 & 0,658545 & 0 \\
\hline Fev 2011 & 138,44 & 53,30 & 0 & 1,15503 & 0 \\
\hline Mar 2011 & 311,38 & 10,80 & 0 & 0,80321 & 0 \\
\hline Abr 2011 & 199,46 & 55,50 & 0 & 0,52873 & 0 \\
\hline Mai 2011 & 26,75 & 0,80 & 0 & 0,002325 & 0 \\
\hline Jun 2011 & 35,15 & 10,80 & 0 & 0,276205 & 0 \\
\hline Jul 2011 & 28,91 & 0,60 & 0 & 0,0009 & 0 \\
\hline Total & 5108,74 & 1244,22 & 15,27 & 29,54 & 0,0085 \\
\hline Média & 170,29 & 41,47 & 0,52 & 0,98 & 0,0003 \\
\hline Desvio Padrão & 144,92 & 50,70 & 2,73 & 1,58 & 0,0011 \\
\hline $\begin{array}{l}\text { Coeficiente de } \\
\text { Variação }\end{array}$ & 0,85 & 1,22 & 5,23 & 1,60 & 3,85 \\
\hline
\end{tabular}


pousio, desenvolvimento da vegetação e adensamento da vegetação). Verifica-se que o sistema de pousio (PO) apresenta perdas de água e solo apenas no período pós-queimada/início do pousio. Após o segundo ano de monitoramento, quando a vegetação do sistema de pousio começa a se desenvolver, há perdas de água inferiores a $1 \mathrm{l}$ ou nulas. 0 manejo convencional, sistema sem cobertura vegetal (SC), apresenta perdas de água e solo ao longo de todo o período de monitoramento. Os valores de escoamento superficial aumentaram no segundo ano em torno de $21 \%$, mesmo com uma significativa diminuição no total pluviométrico e as perdas de solo da parcela SC apresentaram valores de 6,41 t/ha.

Dados similares foram encontrados por E. L. Thomaz (2013), que verificou que a erosão decresceu, a partir da regeneração do sistema. 0 autor constatou também que, após cinco anos de regeneração, a redução da perda de solo foi consideravelmente mais significativa, se comparada com o a redução do escoamento superficial, apresentando uma perda de solo de apenas 0,10 t/ha. Percebe-se que as perdas de solo para o sistema PO decrescem ao longo do tempo o que indica a influência do manejo de pousio na diminuição dos processos erosivos.

Vários estudos têm demonstrado, para áreas abandonadas de corte/queima com pousio, uma redução dos sedimentos e águas disponíveis associadas ao aumento da revegetação natural (M. P. Errea et al., 2001; T. M. Lasanta, S. Bergueria e J. M. Garcia-Ruiz, 2006; Chaves, 2009; E. L. Thomaz, 2013), demonstrando a capacidade do sistema de minimizar os processos erosivos. 0 rápido crescimento da vegetação tropical é um parâmetro importante no restabelecimento da infiltração do solo e na redução da erosão (A. D. Ziegler et al., 2009; E. L. Thomaz, 2013). A partir da regeneração do sistema, ocorrem modificações hidrológicas com a formação de uma camada de manta morta nos primeiros centímetros do solo que terá grande importância na infiltração da água no topo do solo. Além disso, a quantidade de precipitação interna tende a diminuir com o avanço da sucessão, devido à modificação do dossel florestal, que se torna mais alto e estruturalmente mais complexo, aumentando, assim, a interceptação.

\section{Conclusões}

A área de agricultura tradicional (slash-and-burn) apresentou uma boa recarga e drenagem da matriz do solo quando comparada com o sistema sem cobertura vegetal, mostrando-se eficiente no que diz respeito à dinâmica da água em áreas de encostas íngremes. A prática de manejo da agricultura tradicional impõe mudanças nas propriedades dos solos que leva em geral a uma melhoria da qualidade física do solo e causa modificações no balanço hídrico. Percebe-se que as perdas de solo e escoamento superficial para o sistema PO, após cinco anos de regeneração, decresceram 0,008 t/h e 15,2 $\mathrm{mm}$, para valores insignificantes ao longo do tempo, o que indica a influência positiva do manejo de pousio na diminuição dos processos erosivos. Já no sistema SC observou-se que houve um acréscimo nos valores de escoamento superficial no segundo ano de $21 \%$. o uso e o manejo do solo determinam os potenciais matriciais da água no solo, sendo que a área com pousio de 3-5 anos têm um positivo efeito no controle de escoamento superficial e erosão dos solos agrícolas.

\section{Agradecimentos}

Esta pesquisa teve financiamentos do Conselho Nacional de Desevolvimento Científico e Tecnológico (CNPq), processo $n^{\circ}$ 483495/2013-1, Fundação de Amparo à Pesquisa do Estado do Rio de Janeiro (FAPERJ), processos no E-26/111.548/2011, E-26/111.330/2013 e a bolsa de Mestrado de Isabel Linhares Pereira Soares E-26/100.728/2014. À Coordenação de Aperfeiçoamento de Pessoal de Nível Superior (CAPES), pela bolsa de Estágio Sênior no Exterior, nº processo BEX 3959-13-9.

QUADRO III - Escoamento superficial e perda de solo de acordo com as fases de regeneração da agricultura de corte e queima, comparando o período de pousio (PO) com o manejo convencional, sistema sem cobertura vegetal (SC).

$T_{A B L E}$ III - Runoff and soil loss in accordance with the regeneration phases of slash-and-burn agriculture, comparing the fallow period with uncovered soil system.

\begin{tabular}{|c|c|c|c|c|c|}
\hline \multirow{2}{*}{ Período } & \multirow{2}{*}{ Pluviosidade (mm) } & \multicolumn{2}{|c|}{ Escoamento (mm) } & \multicolumn{2}{|c|}{ Perda de solo (t/ha) } \\
\cline { 3 - 6 } & & SC & PO & SC & PO \\
\hline Pós-queimada/Início do pousio & 2428,40 & 462,30 & 15,27 & 19,71080 & 0,00849 \\
(dez. 2008 - dez. 2009) & & & & 0 & 0 \\
\hline $\begin{array}{c}\text { Desenvolvimento da vegetação } \\
\text { (jan. 2010 - dez. 2010) }\end{array}$ & 1608,20 & 559,22 & 6,41287 & 0 \\
\hline $\begin{array}{c}\text { Adensamento da vegetação } \\
\text { (jan. 2011 - jul. 2011) }\end{array}$ & 1072,14 & 222,70 & 0 & 3,42495 & 0 \\
\hline
\end{tabular}


Referências bibliográficas

Barker, G. (2006). The agricultural revolution in prehistory: why did foragers become famers? Oxford, UK and New York, NY: Oxford University Press.

Bertolino, A. V. F. A., Bertolino, L. C. (2010). Agricultura migratória e seus efeitos sobre o solo. In: Agricultores e Território: Práticas e Saberes. $1^{a}$. ed. Rio de Janeiro: Trasso Comunicação, v.1, p. 51-72.

Borggard, O.K., Gafur, A., Petersen, L. (2003). Sustainability appraisal of shifting cultivation in the Chittagong Hill Tracts of Bangladesh. AMBIO: J. Hum Environ. 32, p. 118-123.

Brady, N. C. (1996). Alternatives to slash-and-burn: a global imperative. Agriculture, Ecosystems and Environment, v. 58, $\mathrm{n}^{\circ}$ 1, p.3-11.

Carvalho Filho, A., Lumbreras, J. F., Santos, R. D. (2000). Os Solos do Estado do Rio de Janeiro. In: Silva, L. C. Estudo Geoambiental do Estado do Rio de Janeiro, Brasília, $2^{\text {a }}$ Ed; CD Rom (anexos)

Cerdà, A., Lasanta, T., (2005). Long-term erosional responses after fire in the Central Sapanish Pyrenees. 1, Water and sediment yeld, Catena 60 , p. $59-80$.

Certini, G. (2005). Fire effects on soil system functioning: new insights and future challenge, IJWF 14, p. 339-342.

Chidumayo, E.N., Kwibisa, L. (2003). Effects of deforestation on grass biomas and soil nutrientes satatus in miombo woodland, Zambia, Agriculture, Ecosystems and Environment, v.96, $\mathrm{n}^{\circ} 1, \mathrm{p} .97-105$.

Chaves, M.V.S., (2009). Análise da precipitação interna em fragmentos de Mata Atlântica em diferentes estágios sucessionais submetidos à pousio na APA de Macaé de Cima - Nova Friburgo/RJ (Monografia). São Gonçalo: DGEO FFP/UERJ. 102p.

Costa, K.K.S. (2012). Agricultura Itinerante na APA de Macaé de Cima, Nova Friburgo/RJ: possibilidades legais a partir da análise da vegetação (Monografia). São Gonçalo: DGEO FFP/UERJ. $110 \mathrm{p}$.

Dantas, M. E. (2000). Geomorfologia do Estado do Rio de Janeiro. In: Silva, L. C. Estudo Geoambiental do Estado do Rio de Janeiro. CPRM-Brasília. $2^{\mathrm{a}}$ Ed. 63p. CD Rom (anexos).

DeBano, I.F., (2000). The role of fire and soil heating on water repellency in wildland environments: a review, Journal of Hydrology 231-232, p. 195-206.
Eldredge, E. P., Shock, C. C., Stieber, T. D. (1993). Calibration of granular matrix sensors for irrigation management. Agron. J. 85, p. 1228-1232.

Errea, M. P., Lasanta, T., Ortigosa, L., Cerdà, A. (2001). Soil moisture changes after land abandonment in the Central Spanish Pyrenees, Cuardernos de Investigación Geográfica, 27, p. 47-60.

Filho, A. A. R., Adams, C., Sereni, R. S. (2013). The impacts of shifting cultivation on tropical forest soil: a review. Bol. Mus. Para. Emilio Goeldi, Ciências Humanas, Belém, v. 8, n. 3, p.693-727, set-dez.

FOOD AND AGRICULTURE ORGANIZATION (FAO) (1985). The Tropical Forestry Action Plan. Rome: UN Food and Agricultural Organization.

Frizano, J., Vann, D. R., Jonhson, A. H., Jonhson, C. M., Vieira, I. C. G., Zarin, D. J. (2003). Labile phosphorus in soils of forest fallows and primary forests in the Bragantine Region, Brazil. Biotropica, v. 35, p. 2-11.

Heilbron, M., Pedrosa-Soares, A. C., Campos Neto, M., Silva, L. C., Trouw, R. A. J., Janasi, V. C. (2004). Brasiliano Belts in SE Brazil. Journal of Virtual Explorer, Volume 17, 2004. Disponível em http: / /www. virtualexplorer.com.au/ journal/2004/17. Acesso em: 2014.

Hudson, N. W. (1993). Field measurement of soil erosion and runoff. Soils Bulletin 68, FAO, Rome.

INSTITUTO NACIONAL DE METEOROLOGIA (2014). Normais climatológicas, 1961-1990. Disponível em: http://www.inmet.gov.br/html/clima/ mapas/?mapa=tmax. Acesso em 2014.

Jonhson, C. M., Vieira, I. C. G., Zarin, D. J., Frizano, J., Johnson, A. H. (2001). Carbon and nutrient storage in primary and secondary forests in eastern Amazonia. Forest Ecology and Management, v. 147, n 2, p. 245-252.

Lasanta, T. M., García-Ruiz J. M., Berguería S. (2006). Geomorphic and Hydrological Effects of Traditional Shifting Agriculture in a Mediterranean Mountains Area, Central Spanich Pyrenees. International Mountain Society.

Lawrence, D., D’odorico, P., Diekman, L., Delonge, M., Rishiraj, D., Eaton, J. (2007). Ecological feedbacks following deforestation create the potential for a catastrophic ecosystem shift in tropical dry forest. PNAS, December 26, vol.14, no 52.

Mataix-Solera, J., Cerdà, A., Arcenegui, V., Jordán, A., Zavala, L. M. (2011). Fire effects on soil aggregation: A review, Earth-Science Reviews 109 , p. $44-60$. 
McDonald, M. A., Healey, J. R., Stevens, P. A. (2000). The effects of secondary forest clearance and subsequent land-use on erosion losses and soil properties in the Blue Mountains of Jamaica. Agriculture, Ecosystems and Environment, v. 92, p. 1-19.

Mcgrath, D. G. (1987). The role of biomes in shifting cultivation. HumanEcology, 15, vol. 2, p. 221-242.

Merat, G.(2014). Análise da dinâmica da paisagem sob utilização de coivara em Bioma de Mata Atlântica - Estação Experimental de Pesquisa de Erosão em São Pedro da Serra Nova Friburgo (Dissertação de Mestrado). São Gonçalo: DGEO FFP/UERJ, 153 p.

Mertz, O., Padoch, C., Fox, J., Cramb, R. A., Leisz, S.J., Lam, N. T., Vien, T. D. (2009). Swidden change in Southeast Asia: understanding causes and consequences. Hum. Ecol. 37, p. 259-264.

Night, R., Diemont, S. A. W. (2013). The Mya milpa: fire and the legacy of living soil. The Ecological Society of America Journal, Ecol Environ. Online Special Issue: Prescribed burning, p. 45-54.

Nye, P. H., Greenland, D. J., (1960). The soil under shifting cultivation. London, UK:CAB.

Obale-Ebanga, F., Sevink, J., de Groot, W., Nolte, C. (2003). Myths of slssh and burn on physical degradation of savannah soils: impacts on Vertisols in north Cameroon, Soil Use Manage. 19, p. 83-86.

Padoch, C., Pinedo-Vasquez, M. (2010). Saving slash-andburn to save biodiversity. Biotropica, v. 42, n. 5, p. 550-552.

Pedroso Júnior, N.N, Murrieta, R.S.S., Adams, C. (2009). Slash-and-burn agriculture: a system in transformation: In: Begossi, A., Lopes , P. (Orgs.) Current trends in human ecology. Cambridge: Cambridge Scholars Publishing, p.12-34.

Pedroso Júnior, N.N, Murrieta, R.S.S., Adams, C. (2008). A agricultura de corte e queima: um sistema em transformação. Bol. Mus. Para. Emilio Goeldi, Ciências Humanas, Belém, v. 3 n. 2, p.153-174, maio-ago.

Ross, J. L. S., (1985). Relevo Brasileiro: uma nova proposta de classificação. Revista do Departamento de Geografia, São Paulo, v.4, p. 25-39.

Sanchez, P. A., Palm, C. H., Vosti, S. A., Tomich, T. P., Kasyoki, J., (2005). Alternatives to slashand-burn: Chanllenge and approaches of an
International Consortium. In: Palm, C. H., Vosti, S. A., Sanchez, P. A., Polly, J. E. (Eds.) Slash-andburn agriculture: the search for alternatives. New York: Columbia University Press.

Shakesby, R., Doerr, S. (2006). Wildfire as a hydrological and geomorphological agent. Earth-Sci. Rev. 74, p. 269-307.

Shock, C. C. (2003). Soil water potential measurement by granular matrix sensors. In: B. A. Stewart and T.A. Howell (Editors), Encyclopedia of Water Sciences. Marcel Dekker, pp. 899-903.

Shock, C. C., Brnum, J. M., Seddigh, M. (1998). Calibration of watermark soil moisture sensors for irrigation management. Int. Irrig. Show, San Diego, CA, USA, p. 139-146.

Styger, E. R., Harivelo, M., Pfeffer, M.J., Fernandes, E.C.M., Bates, D.M., (2007). Influence of slsah-and-burn farming practices on fallow succession and land degradation in the rainforest region of MAdagascar, Agriculture, Ecosystems e Environment, v. 119, n 3-4, p. 257-269.

Thomaz, E. L. (2013). Slash-and-burn agriculture: establishing scenarios of runoff and soil loss for a Five-year cycle, Agriculture, Ecosystems and Environment 168, p. 1-6.

Thornthwaite, C. W., Mether, J. R. (1955). The water balance. Centerton, NJ: Drexel Institute Of Technology Laboratory of Climatology. Publications in Climatology, vol.VIII, $\mathrm{n}^{0} 1$, $104 \mathrm{p}$.

Valverde, O. (1968). Sistemas de roça (agricultura nômade ou itinerante). Finisterra, volume III, 6, p. 225-239.

Vomocil, J. A., Flocker, W. J., (1966). Effects of soil compaction on storage and movement of soil, air, water, Transactions American Society of Agricultural Engineers 4, p. 242-246.

Warkentin, B. P. (1971). Effects of compaction on content and transmission of water in soils. In: Barnes, K. (Ed.). Compaction of Agricultural Soils. ASAE, p. 125-140.

Ziegler, A. D., Bruun, T. B., Guardiola-claramonte, M., Giambelluca, T. W., Lawrence, D., Thanh Lan N., (2009). Environmental consequences of the demise in swidden cultivation in Montane rainfall Southeast Asia: hydrology and geomorphology, Hum Ecol., p. 37, 36. 